\section{SMARTPHONE REMINDER FOR PHYSICAL ACTIVITY IN PEOPLE WITH INTELLECTUAL DISABILITIES}

doi:10.1017/50266462317000630

\section{BACKGROUND}

Several studies have been carried out regarding different interventions in an effort to improve levels of physical activity in people with an intellectual disability (ID). Studies have been carried out regarding physical activity interventions (1), educational interventions (2), and multimodal interventions (3). New technologies are currently being used to carry out changes in daily habits and to promote health in different pathologies but not in people with an ID $(4 ; 5)$. Due to the poor maintenance of physical activity that we have found in people with ID, the objective of the present study is to determine the maintenance of levels of physical activity, quality of life, self-efficacy, and social support in this population through an intervention with an application in their smartphones.

\section{METHODS}

A randomized controlled trial was carried out. Eight people with mild ID (intelligence quotient $=55-70$ ) participated at the occupational center, Aspromanis Industrial (Malaga, Spain). All participants carried out a multimodal intervention (physical activity + education) (6). After the intervention, the sample was divided in two groups: four people with the smartphone intervention and four in the group with no intervention. The smartphone intervention consisted of installing an application in their smartphones. With this application, educational advice

The authors are grateful to the Dr. Maria Jose Moron and Dr. Rafael Luque who develop the App advice4health and the volunteers for their participation in Aspromanis Industrial, Malaga (Spain). The authors declare that there is no source of funding regarding the publication of this study. and reminders appeared in their smartphones every 2 days. The smartphone intervention was carried out over a 12-week period. People in the smartphone intervention group had to answer at least 80 percent of the messages or their data were considered as drop-outs. Outcome measures (International Physical Activity Questionnaire [IPAQ], WHOQoL, and SelfEfficacy/Social Support Scales for Activity for persons with Intellectual Disability [SE/SS-AID]) were measured before and after the intervention. Physical activity was measured in metabolic equivalent of task (METs) as the amount of oxygen consumed while sitting at rest and is equal to $3-5 \mathrm{ml} \mathrm{O}_{2}$ per $\mathrm{kg}$ body weight $\times \min (7)$.

\section{RESULTS}

In the present study, significant intragroup increase were found in the Smartphone group in vigorous physical activity $(F=$ $8.21 ; p=.01)$, METS walking $(F=13.61 ; p=.02)$, and total METS $(F=6.74 ; p=.05)$. Changes found between both groups in quality of life $(F=0.23 ; p=.01)$, professional support $(F=6.72 ; p=.04)$, and peer support $(F=8.33 ; p=.03)$ are shown in Table 1.

\section{DISCUSSION}

In the literature, we have not found any similar studies on a smartphone intervention for people to encourage physical activity in people with an ID, so this is the first study of this kind. Similar interventions that have used a smartphone application to serve as reminders have been carried out among people with cardiac diseases $(8)$ and among healthy people $(9 ; 10)$. The main strength of the present pilot study is the novelty of the use of an application for smartphones among people with ID because no previous studies had been conducted using a similar intervention in this population, and it is very important to know the changes that new technologies could offer to this population. However, it would be important to carry out this intervention 
Table 1. Differences after Smartphone Intervention

\begin{tabular}{lrcccc}
\hline & & $\begin{array}{c}\text { Smartphone group } \\
\text { Mean (SD) }\end{array}$ & $\begin{array}{c}\text { Control group } \\
\text { Mean (SD) }\end{array}$ & $\begin{array}{c}\text { Intragroup } \\
\text { differences F(Sig) }\end{array}$ & $\begin{array}{c}\text { Intergroup } \\
\text { differences F(Sig) }\end{array}$ \\
\hline METS VIG & Pre & $4000( \pm 4928.20)$ & $4320( \pm 6668.37)$ & $0.01(0.92)$ & $2.68(0.13)$ \\
& Post & $5600( \pm 9699.48)$ & $2335.00( \pm 3256.22)$ & $8.21(0.01)$ & \\
METS MOD & Pre & $320( \pm 277.13)$ & $480( \pm 157.27)$ & $0.40(0.56)$ & $1.25(0.29)$ \\
& Post & $880( \pm 603.99)$ & $200( \pm 69.28)$ & $4.53(0.10)$ & \\
METS WALKING & Pre & $825( \pm 644.13)$ & $462( \pm 457.26)$ & $0.16(0.71)$ & $12.17(0.01)$ \\
& Post & $511.5( \pm 23.93)$ & $1980( \pm 3086.51)$ & $13.61(0.02)$ & \\
METS TOTAL & Pre & $5145( \pm 6524.64)$ & $5262( \pm 6839.92)$ & $0.18(0.90)$ & $2.71(0.13)$ \\
& Post & $6991.5( \pm 9935.12)$ & $2180( \pm 3121.73)$ & $6.74(0.05)$ & \\
QoL & Pre & $29.00( \pm 2.65)$ & $27.00( \pm 4.58)$ & $0.02(0.55)$ & $3.75(0.05)$ \\
& Post & $30.50( \pm 1.29)$ & $27.00( \pm 1.42)$ & $0.23(0.01)$ & \\
SE & Pre & $1.50( \pm 0.44)$ & $1.53( \pm 0.48)$ & $0.03(0.88)$ & $3.32(0.23)$ \\
& Post & $1.63( \pm 0.72)$ & $1.58( \pm 0.32)$ & $3.17(0.32)$ & \\
SFamily & Pre & $1.78( \pm 1.07)$ & $1.75( \pm 0.81)$ & $0.32(0.59)$ & $2.05(0.17)$ \\
& Post & $1.54( \pm 0.67)$ & $1.17( \pm 0.19)$ & $2.69(0.15)$ & \\
SProf & Pre & $2.61( \pm 0.38)$ & $2.33( \pm 0.75)$ & $0.93(0.37)$ & $1.85(0.08)$ \\
& Post & $2.17( \pm 0.93)$ & $1.29( \pm 0.34)$ & $6.72(0.04)$ & \\
SPeers & Pre & $2.00( \pm 0.72)$ & $2.10( \pm 0.77)$ & $0.17(0.69)$ & $4.22(0.03)$ \\
& Post & $2.15( \pm 0.93)$ & $1.25( \pm 0.30)$ & $8.33(0.03)$ & \\
& & & & &
\end{tabular}

Note. Bold values are made for significant changes.

MET, metabolic equivalent of task; METS VIG, vigorous METS, METS MOD, moderate METS, QoL, quality of life; SE, selfefficacy, SFamily, family support, SProf, professional support; Speers, peers support.

in a bigger sample of participants and during a longer period of time to see if similar changes are found and if they are sustained over a longer period.

\section{CONCLUSIONS}

People with IDs who carried out an intervention with smartphones had greater levels of maintenance of physical activity than did those who did not use this intervention. The intervention with the smartphone also increased quality of life and professional and peer support.

\section{CONFLITS OF INTEREST}

The authors have nothing to disclose.

\section{David Pérez-Cruzado \\ Department of Physiotherapy, University of Malaga \\ Antonio Ignacio Cuesta-Vargas \\ Department of Physiotherapy School of Clinical Science, Faculty of Health Science, Queensland University Technology \\ acuesta@uma.es}

\section{REFERENCES}

1. Mitchell F, Melville C, Stalker K, et al. Walk well: A randomised controlled trial of a walking intervention for adults with intellectual disabilities: Study protocol. BMC Public Health. 2013;13: 620.

2. Heller T, Sorensen A. Promoting healthy aging in adults with developmental disabilities. Dev Disabil Res Rev. 2013;18:22-30.

3. Heller T, McCubbin JA, Drum C, Peterson J. Physical activity and nutrition health promotion interventions: what is working for people with intellectual disabilities? Intellect Dev Disabil. 2011;49:26-36.

4. Sheil F, Davis S, Lowery AJ, Hill ADK. The use and limitations of SMS reminders to improve outpatient attendance rates. 2014;107:189.

5. Patterson V. Telemedicine for epilepsy support in resource-poor settings. Front Public Health. 2014;2:120.

6. Pérez-Cruzado D, Cuesta-Vargas AI. Changes on quality of life, selfefficacy and social support for activities and physical fitness in people with intellectual disabilities through multimodal intervention. Eur J Spec Needs Educ. 2016;0:1-12.

7. Jetté M, Sidney K, Blümchen G. Metabolic equivalents (METS) in exercise testing, exercise prescription, and evaluation of functional capacity. Clin Cardiol. 1990;13:555-565.

8. Antypas K, Wangberg SC. An Internet- and mobile-based tailored intervention to enhance maintenance of physical activity after cardiac rehabilitation: short-term results of a randomized controlled trial. $J$ Med Internet Res. 2014;16:e77.

9. Thorsteinsen K, Vittersø J, Svendsen GB. Increasing physical activity efficiently: an experimental pilot study of a website and mobile phone intervention. Int J Telemed Appl. 2014;2014:746232.

10. Glynn LG, Hayes PS, Casey M, et al. Effectiveness of a smartphone application to promote physical activity in primary care: The SMART MOVE randomised controlled trial. Br J Gen Pract J R Coll Gen Pract. 2014;64:e384-e391. 